\title{
Apercevoir l'amour entre personnes de même genre au Moyen Âge
}

Seeing Sodomy de Robert Mills au sein des études sur l'homosexualité médiévale.

\section{Chloé Clovis Maillet}

\section{CpenEdition}

\section{Journals}

Édition électronique

URL : http://journals.openedition.org/imagesrevues/8296

DOI : 10.4000/imagesrevues.8296

ISSN : $1778-3801$

Éditeur :

Centre d'Histoire et Théorie des Arts, Groupe d'Anthropologie Historique de l'Occident Médiéval Laboratoire d'Anthropologie Sociale, UMR 8210 Anthropologie et Histoire des Mondes Antiques

\section{Référence électronique}

Chloé Clovis Maillet, «Apercevoir l'amour entre personnes de même genre au Moyen Âge », Images Revues [En ligne], 17 | 2020, mis en ligne le 16 septembre 2020, consulté le 30 janvier 2021. URL : http:// journals.openedition.org/imagesrevues/8296 ; DOI : https://doi.org/10.4000/imagesrevues.8296

Ce document a été généré automatiquement le 30 janvier 2021.

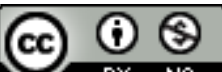

Images Re-vues est mise à disposition selon les termes de la Licence Creative Commons Attribution Pas d'Utilisation Commerciale 4.0 International. 


\section{Apercevoir l'amour entre personnes de même genre au Moyen Âge}

Seeing Sodomy de Robert Mills au sein des études sur l'homosexualité médiévale.

\section{Chloé Clovis Maillet}

1 Que peut-on voir de l'homoérotisme dans l'occident médiéval? Le désir pour les personnes de même genre (comme le désir sexuel en général mais de manière encore plus radicale) était condamné par «l'institution dominante de cette société $»^{1}$, l'Église chrétienne, et cette dernière était aussi une des principales forces de commandes d'images, pour la décoration de ses établissements, pour le culte et les images de ses manuscrits. On serait donc condamnés à un regard oblique, dans les interstices de la création. En croisant des méthodes issues de l'analyse iconographique serielle, de l'étude de la réception et des

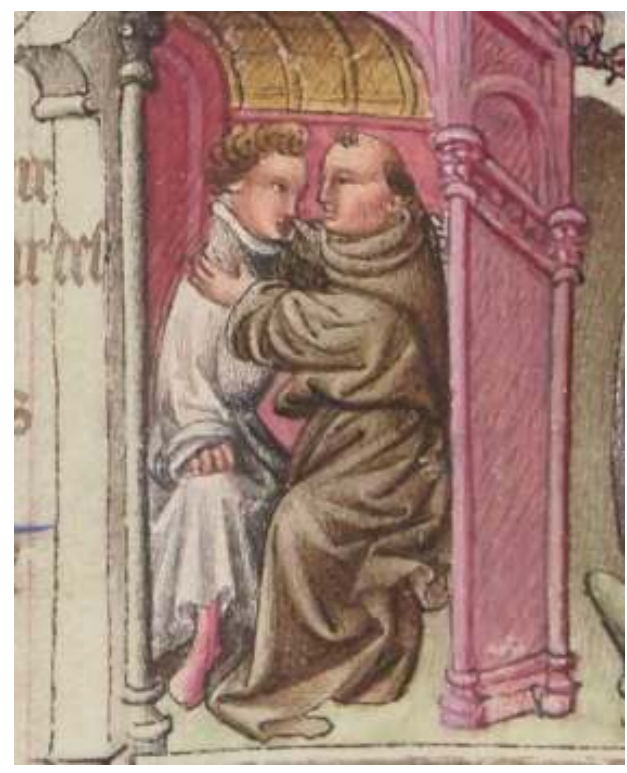
questionnements sur le corps, l'approche radicalement originale de Mills ${ }^{2}$, inédite en langue française, suscite une confrontation stimulante avec le champ des études sur l'homosexualité.

\section{Entre justice et littérature amoureuse}

Le débat sur la question homosexuelle dans le christianisme médiéval est complexe et a occasionné de multiples controverses, opposant de longue date une vision «tolérante » (appuyée sur l'immensité et la sensualité de la littérature de l'amitié virile 
qui faisait le fondement des études de John Boswell ${ }^{3}$ ) à une vision répressive (fondée sur les non moins abondantes condamnations du crime " sodomite ", trop rapidement assimilé à l'homosexualité moderne). Les deux pôles théoriques de l'amour entre personnes de même genre se situent donc entre la criminalisation juridique et la culture amoureuse qui fait une large place à des effusions intenses surtout entre hommes $^{4}$ et parfois entre femmes ${ }^{5}$. Comprendre la description des effusions "amicales" entre chevaliers ou entre moines requiert une immense modestie dans notre capacité à saisir le "secret des alcôves " (Alain Corbin'6), et l'appréhension des changements anthropologiques majeurs qui déterminent l'histoire des sexualités. Comprendre la situation dans le domaine militant permet de saisir certains des enjeux du débat historiographique. John Boswell (1947-1994), initiateur et précurseur dans de multiples domaines aux marges de la médiévistique (l'abandon des enfants, le célibat et l'homosexualité), était un historien militant pour les droits de homosexuels dans cette période tragique des années 1970-1980 touchée par l'épidémie meurtrière du sida. Il se présentait également comme une personne de confession chrétienne soucieuse de réconcilier l'église de son temps avec la communauté gaie. En face, une grande partie des médiévistes de l'époque était dominée par des personnes hétérosexuelles et souvent pratiquantes du christianisme ${ }^{7}$, parfois soucieuses de montrer une continuité entre l'hétéronorme défendue à l'époque moderne par les autorités religieuses et ses origines médiévales. Quelques chercheurs, comme Jean Wirth, se sont distingués par une approche toute aussi érudite qu'irrévérencieuse des questions religieuses, et résolument ouverte à une dimension libertaire des questions sexuelles, n'hésitant pas à chercher dans les cloîtres des églises romanes les traces de la subculture gaie mise en valeur par Boswell, mais on pouvait lui reprocher une vision essentialiste et psychanalytique de l'homosexualité.

3 Jean-Claude Schmitt avait brillamment expliqué les enjeux du problème dans la culture cléricale médiévale dans un compte rendu du livre de Boswell à l'heure où on débattait de la dépénalisation de l'homosexualité en France en 1981 :

Pour l'Église, le véritable ennemi n'est pas l'homosexuel, mais la femme. Alors que la virginité s'impose comme valeur suprême du chrétien, que la chasteté devient règle de vie, que le péché originel est identifié au désir charnel de l'homme et de la femme, l'hostilité vouée à l'homosexualité n'a qu'une importance relative par rapport l'adultère ou l'inceste. Par ailleurs, il est important de garder à l'esprit que ces mêmes Pères de l'église, une minorité très écoutée qui censuraient les conduites homosexuelles, censuraient aussi, et avec autant de sévérité, des comportements qui sont aujourd'hui universellement acceptés par les communautés chrétiennes. Le prêt à intérêt, les rapports sexuels pendant la menstruation, les bijoux et les étoffes teintées, le rasage, les bains réguliers, le port de la perruque, le service militaire ou les fonctions du gouvernement civil, le travail manuel les jours de fêtes religieuses, la nourriture cachère et la circoncision, ont tous été absolument condamnés par différents Pères de l'Église, les mêmes qui, victimes de leurs préjugés, mal renseignés, ou enclins interpréter la Bible dans un sens rigoureusement littéral, condamnaient l'homosexualité et bien d'autres pratiques ${ }^{8}$.

Si l'analyse de Schmitt reste d'actualité, l'approche de John Boswell a depuis été placée parmi les penseurs essentialistes de l'homosexualité, par rapport aux constructionistes qui défendent la contingence socio-historiques des sexualités. L'homosexualité, en tant qu'orientation sexuelle pathologisée au $\mathrm{XIX}^{\mathrm{e}}$ siècle, n'est pas un problème médiéval. Un spécialiste de l'histoire de l'homosexualité antique, David Halperin ${ }^{9}$ préfère parler de pré-homosexualité pour évoquer les périodes pré-modernes, et explique que l'idée 
même d'orientation sexuelle et d'une identité corrélée, n'était pas adaptée pour évoquer ces périodes. Il en va de même pour l'hétérosexualité pensée comme elle l'est aujourd'hui ${ }^{10}$. Les personnes avaient une sexualité faite d'actes successifs dont certains étaient répréhensibles, parfois durement. La demande de faire l'archéologie de ces amours est forte, et le nombre modeste de publications scientifiques sur la question ne peut faire face aux publications opportunistes comme A Gay History of Britain ${ }^{11}$ and A Lesbian History of Britain avec les problèmes téléologiques qui peuvent se poser, et contre lequel se positionne opportunément le livre de Robert Mills (p. 17). C'est tout l'intérêt de son ouvrage que de s'inscrire dans la perspective d'une nouvelle philologie des sexualités médiévales, plus complexes que nos catégories genre/sexualités vouées à mal embrasser les termes médiévaux tel que celui du péché de "sodomie». Les questions de méthodes deviennent alors patentes.

\section{Actes sexuels, fluidité de genre et orientations}

L'histoire de la sexualité de Michel Foucault, qui constitue la référence principale dans ce champ n'aborde quasiment pas la période médiévale, si ce n'est la patristique dans ses écrits posthumes ${ }^{12}$. La question est à la fois d'ordre théorique (qu'est-ce que la sexualité ?) et pratique (on ne dispose presque uniquement que de la patristique à l'époque tardo-antique, l'archéologie n'étant que de peu de ressources, et de sources judiciaires à partir du $\mathrm{XI}^{\mathrm{e}} \mathrm{s}$ ). John Boswell, qui écrivait en même temps que Foucault, avait opté pour une historicisation précise : tolérance de tradition tardo-antique et amitié amoureuse jusque vers 1150, date de parution du Liber Gomorrhianus de Pierre Damien fondant le "crime contre-nature» comme une hérésie. Damien Boquet, spécialiste d'Aelred de Rielvaux optait plutôt pour une perspective qui voudrait que coexistent sur un temps long (mais sans remettre en question le jalon du XII ${ }^{\mathrm{e}}$ siècle) littérature de l'amour (entre personnes de même sexe) et répression des actes dans des champs sociaux et culturels différents ${ }^{13}$.

6 Le livre de Robert Mills propose l'usage d'un concept endogène: le "péché de sodomie ». Ce péché fait l'objet de définitions variables qui englobent notamment, mais pas seulement, celui de l'amour physique entre personnes de même genre. Mills se sert de l'exemple d'une miniature des Belles Heures du duc de Berry montrant Jérôme moqué par les moines parce qu'il porte une robe féminine, pour déconstruire un certain nombre de clichés portant sur l'association entre transgression de genre et orientation sexuelle. Le récit, inspiré de la Légende dorée, évoque le moment où la grande popularité de Jérôme suscite des jalousies et un moine dépose un habit féminin dans sa cellule pour le faire accuser d'adultère. Jérôme se lève à l'aube pour la prière et enfile la robe en question. Les moqueries qu'il attire sont le point de départ de son choix de rejoindre le désert. Pourtant ces moqueries n'ont rien à voir avec la suspicion d'efféminement homosexuel du théologien. Elles proviennent du ridicule de la mise, un habit de femme et donc non convenable pour son statut. Les moqueries, ajouterais-je à l'analyse de Mills sont aussi liées au fait que Jérôme soit dans cette image mal habillé, d'une cottardie portée sans chainse dont la mise est elle-même ridicule, alors que Jérôme était réputé fondateur de la moralisation du costume et de l'adhésion entre intériorité et apparence. Et même l'écriture de ce récit daterait du Moyen Âge central, elle pouvait s'appuyer sur les reproches fait à la trop grande attention que Jérôme avait pour les femmes saintes ${ }^{14}$. Si Mills évoque la possibilité que les allusions sexuelles présentes 
dans les Belles heures soient à mettre en lien avec les mœurs sexuelles réputées dépravées de Jean de Berry (et même une accusation de sodomie ${ }^{15}$ ) ce n'est sans doute pas tant cette image qui en témoigne que les multiples scènes de tentations féminines, suffisamment explicites dans le recueil. S'il est question de sexualité dans cette image, c'est bien de chasteté, définie par Ruth Mazo Karras ${ }^{16}$ comme une forme de sexualité (au sommet de la hiérarchie des pratiques sexuelles). L'asexualité est aujourd'hui revendiquée par une minorité de personnes dans un contexte bien différent, en suivant des arguments déconnectés des revendications morales qui en faisaient l'honneur au Moyen Âge. À l'époque médiévale, l'abstinence sexuelle permettait une certaine fluidité dans les présentations et revendications de genre, que Mills lit avec un «regard transgenre ». Dans plusieurs cas, celui de la recluse Christina de Markyate, un temps vêtue d'un habit masculin (chapitre 5), de sainte Eugénie, ayant pris l'identité d'un moine, accusé d'adultère (chapitre 2), ou de Jérôme de Stridon malencontreusement habillé en femme (introduction), la tension confronte pratique sexuelle et abstinence, non celle d'une orientation homo ou hétérosexuelle.

Fig. 1

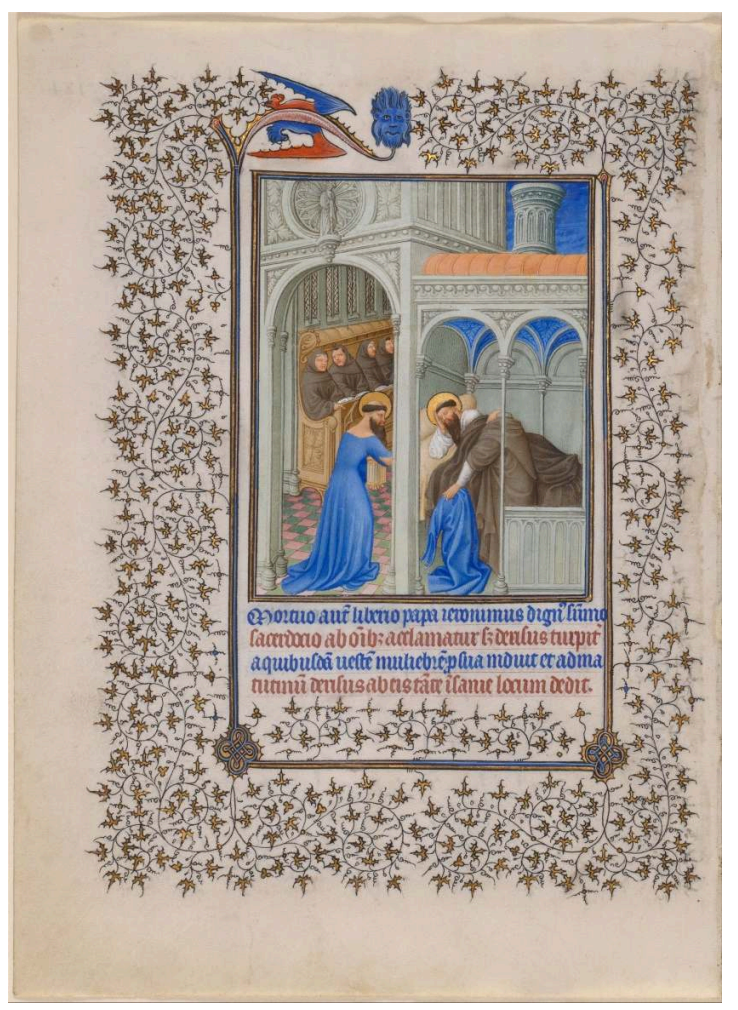

Un moine déposant un vêtement féminin sur le lit de Jérôme de Stridon, Jérôme portant ce vêtement à la prière du matin, une lanterne à la main. Les frères de Limbourg, Les Belles Heures du duc de Berry, $\mathrm{f}$. 184v. 1405-1409. Metropolitan Museum of Art, The Cloisters collection, 54.1.1a, domaine public CCO

7 La théorisation médiévale la plus précise avancée pour déterminer le péché de sodomie émane de Pierre Damien ${ }^{17}$. Or ce texte pose divers problèmes. Il ne concerne que les hommes et se désintéresse des femmes, et considère comme appartenant au péché sodomite l'autopollution (masturbation autosexuelle), le frottage des virilia (masturbation), les rapports intercruraux (entre les cuisses), et la fornication in terga (rapport anal) ${ }^{18}$. Didier Lett a pu montrer la persistance de la polysémie du terme "sodomie » par une étude précise des sources judiciaires à Bologne au $\mathrm{Xv}^{\mathrm{e}}$ siècle : à 
cette époque le vice sodomite servait essentiellement à condamner ce que nous désignerons aujourd'hui comme des actes de pédocriminalité, entre un adulte et un enfant non consentant ${ }^{19}$. Robert Mills évoque une image d'une Bible moralisée, des mêmes frères Limbourg qui présente les habitants de Sodome comme couples entrelacés, de même genre mais aussi de genre différents, troublant la fausse familiarité que nous pensons avoir avec le comportement sexuel des pécheurs bibliques. Voir la Sodome médiévale signifie se poser des questions plus générales sur le genre et les sexualités et la manière dont elles se donnaient à voir à cette époque. Sa position s'oppose fortement avec celle d'un Jean Wirth qui présentait les gomorrhéens de Pierre Damien exactement comme nous parlerions de couples homosexuels aujourd'hui ${ }^{20}$.

Fig. 2

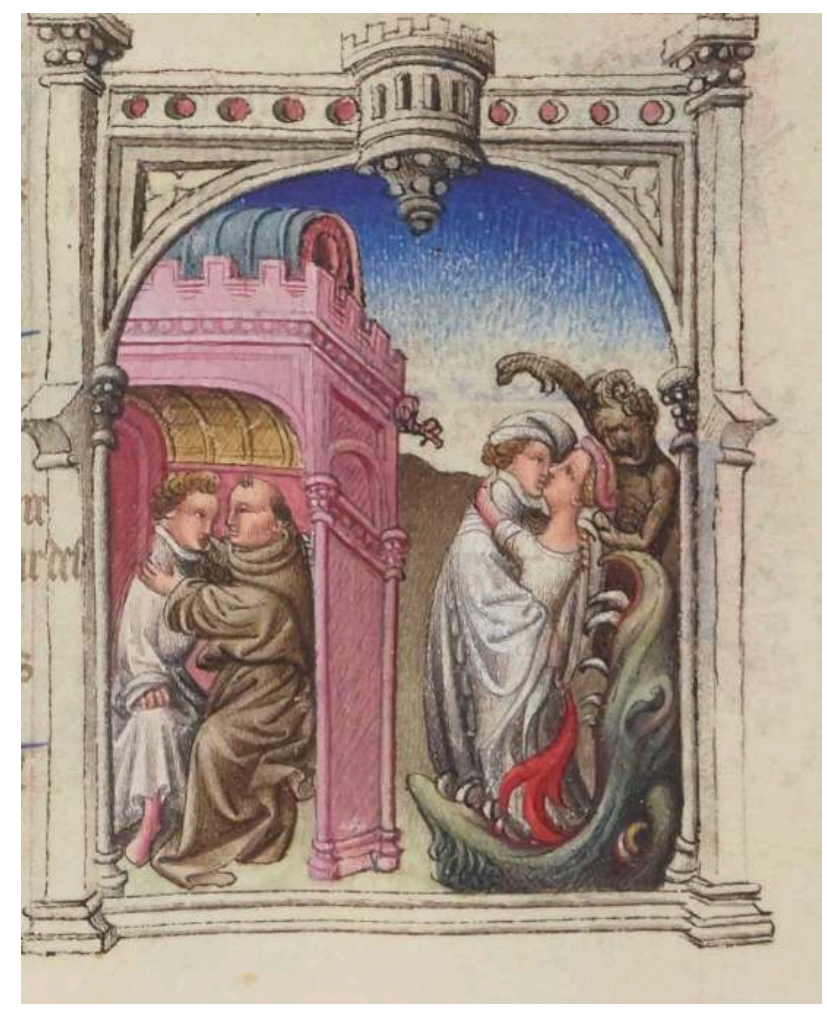

Les frères de Limbourg, Bible moralisée, Paris, Bibliothèque nationale de France, ms français 166, fol. $7 r$.

Pourtant, comme l'avait bien démontré Ruth Mazo Karras, la sexualité était alors pensée comme une série discontinue d'actes répréhensibles ou non, faits "par une personne sur une autre $»^{21}$. Ce qui entraine une distinction fondamentale en termes de peines en fonction de la personne actrice, considérée comme active dans cet acte. Aussi la sodomie entre femmes était réprimée seulement en cas d'usage d'objets permettant l'acte ainsi défini, et donc a laissé moins de traces de répression les $\operatorname{archives}^{22}$, mais bien mentionné dans les pénitentiels :

As-tu fait comme ces femmes qui pour étreindre le désir qui les tourmente se joignent comme si elles pouvaient s'unir ? As-tu fait comme certaines femmes ont coutume de faire, as-tu fabriqué une machine de la taille qui te convient, l'as-tu lié à l'emplacement de ton sexe ou de celui d'une compagne et as-tu forniqué avec 
d'autres mauvaises femmes ou d'autres avec toi, avec cet instrument ou un autre Burchard de Worms (XI ${ }^{\mathrm{e}}$ siècle $)^{23}$.

9 Mills, loin de chercher dans les images une illustration des sexualités, propose un pas de côté qui vise à comprendre ce que signifie le fait de regarder un phénomène défini sous le terme de «sodomie ». Il étudie le corpus iconographique explicitement lié à la question de la sodomie, celui de la destruction de Sodome dans la Bible (chap 1), et montre l'interconnexion entre sexualités et genre (et temporalité transgenre en suivant J. Halberstam ${ }^{24}$ ) (chap 2). Il propose Orphée, modèle du premier sodomite des images, comme modèle du concept queer d'échec (chap 3). Dans un chapitre sur la sexualité des moines, et sans éluder les douloureuses questions de pédocriminalité au sens de l'église, il détaille l'ambivalence de l'enlèvement de Ganymède, tantôt châtiment divin effrayant le jeune pécheur, tantôt moralisé comme union avec la divinité. Le dernier chapitre propose la virginité comme une forme de sexualité (chez Christina de Markyate, encore marquée par la transidentité). Le livre se conclut sur la "phénoménologie de l'anus» omniprésente à la fois dans les marginalia, et dans certaines visions de l'enfer.

On pourrait reprocher certaines disjonctions au plan de son ouvrage en partie dues au fait que certains chapitres avaient été précédemment publiés dans d'autres contextes ${ }^{25}$. Mais son intérêt est d'éviter à tout prix une forme de catalogage des types d'images qui permettent d'aborder la question. Aussi en entrelaçant question de genre et question de sexualité, Mills montre la plasticité de la question de la sodomie. Il manque cependant à l'ouvrage le traitement des sexualités non reproductrices comme l'onanisme et l'animalité, qui étaient alors englobées dans ce concept.

\section{Le péché de Sodome}


Fig. 3

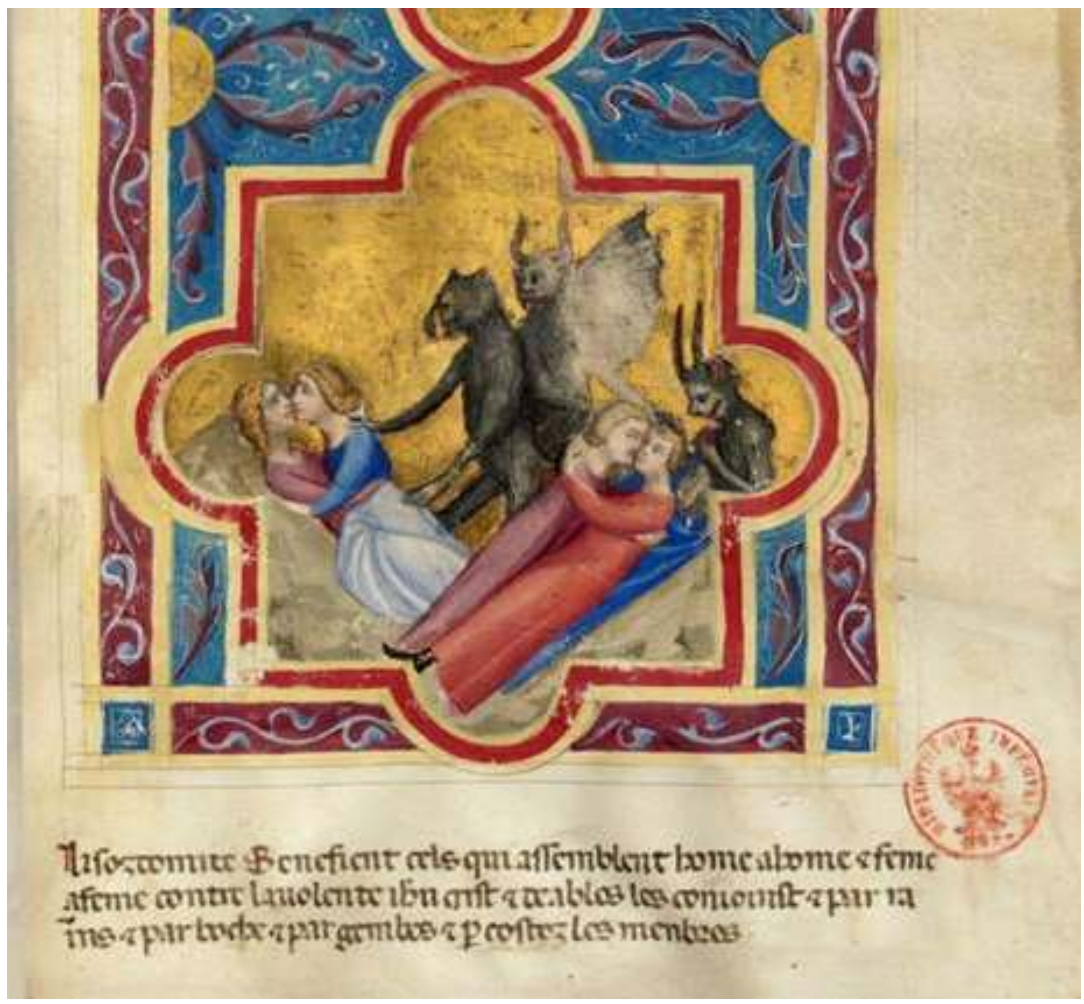

Bible, BnF Français 9561, f. 17., c. 1350.

$\mathrm{BnF}$

11 Dans les bibles moralisées, finement analysées, les péchés sexuels sont variés, mais tendent à se fixer autour de la présentation de couples enlacés, d'abord l'un masculin, l'autre féminin (dans les Bibles de Vienne et celle de la $\mathrm{BnF}$ ), puis uniquement masculins. Les couples de femmes «se meuvent dans le flou selon les époques » (p. 64) sans qu'il soit évident de comprendre si leur présence ou absence est due davantage à la transmission d'un manuscrit à l'autre ou à l'intérêt de l'image. Ces images d'étreintes impressionnent parce qu'elles semblent montrer sans voile des gestes d'amour entre hommes ou entre femmes. Parce qu'explicites pour nos contemporains, elles sont devenues des images de référence pour culture homosexuelle contemporaine. Pourtant, ces images n'étaient certainement pas des images positives d'auto-identification à l'époque, puisqu'elles présentaient des personnages négatifs et condamnés moralement, et destinés aux pires châtiments. 
Fig. 4

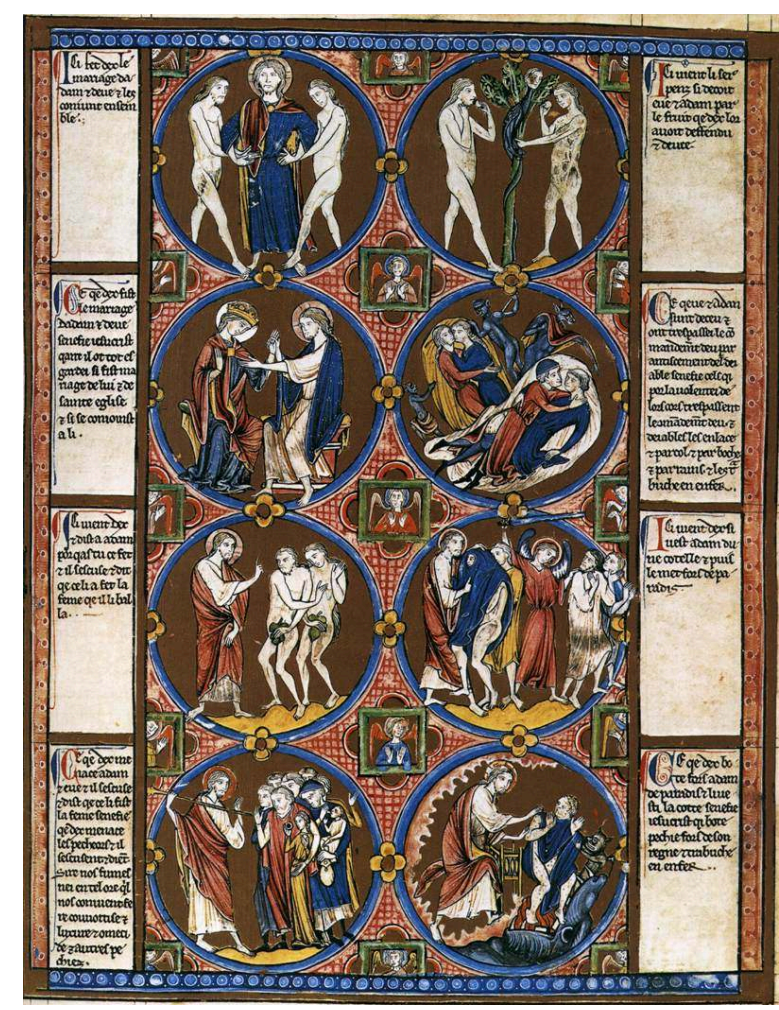

Vienna, Osterreichische National Bibliothek, cod 2554, fol 2 r.

12 Chercher à comprendre la question, ce n'est donc pas se concentrer pour chercher une culture homosexuelle sous l'image de ces couples opprimés, c'est penser plus largement les flous, les transitions la fluidité des genres et de l'amour. Mills use du terme de translatio généralement pensé pour désigner le transitus, le passage d'un monde à l'autre, afin de saisir l'anthropologie médiévale des sexualités. Désigner une femme jouant un rôle d'homme dans l'acte de chair, est sous la plume d'Hildegarde de Bingen ce qu'il y a de plus vil (vilissima in conspectu meo), parce qu'il y a une usurpation de droit et de statut, celui d'homme dans lequel elles se transmutent (se transmutaverunt) (Hildegarde de Bingen, Scivias, 279, 42, cité p. 94). 
Fig. 5

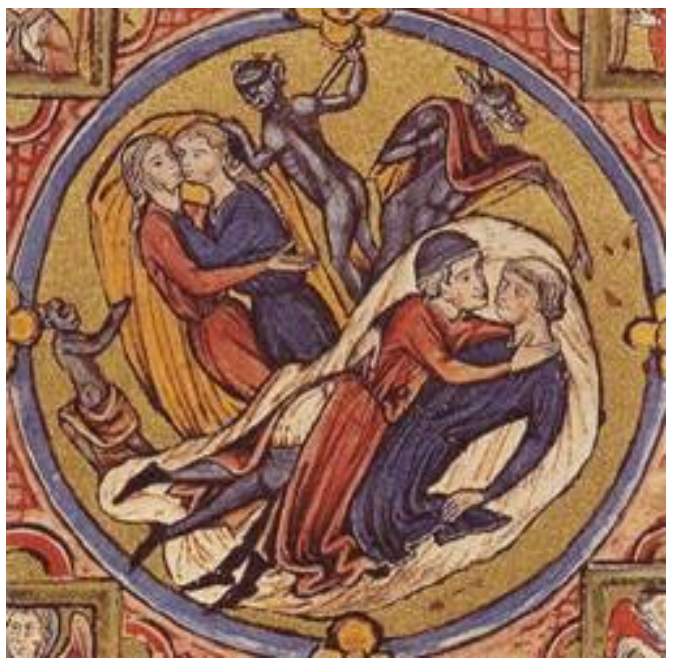

Vienna, Osterreichische National Bibliothek, cod 2554, fol 2 r., détail

Fig. 6

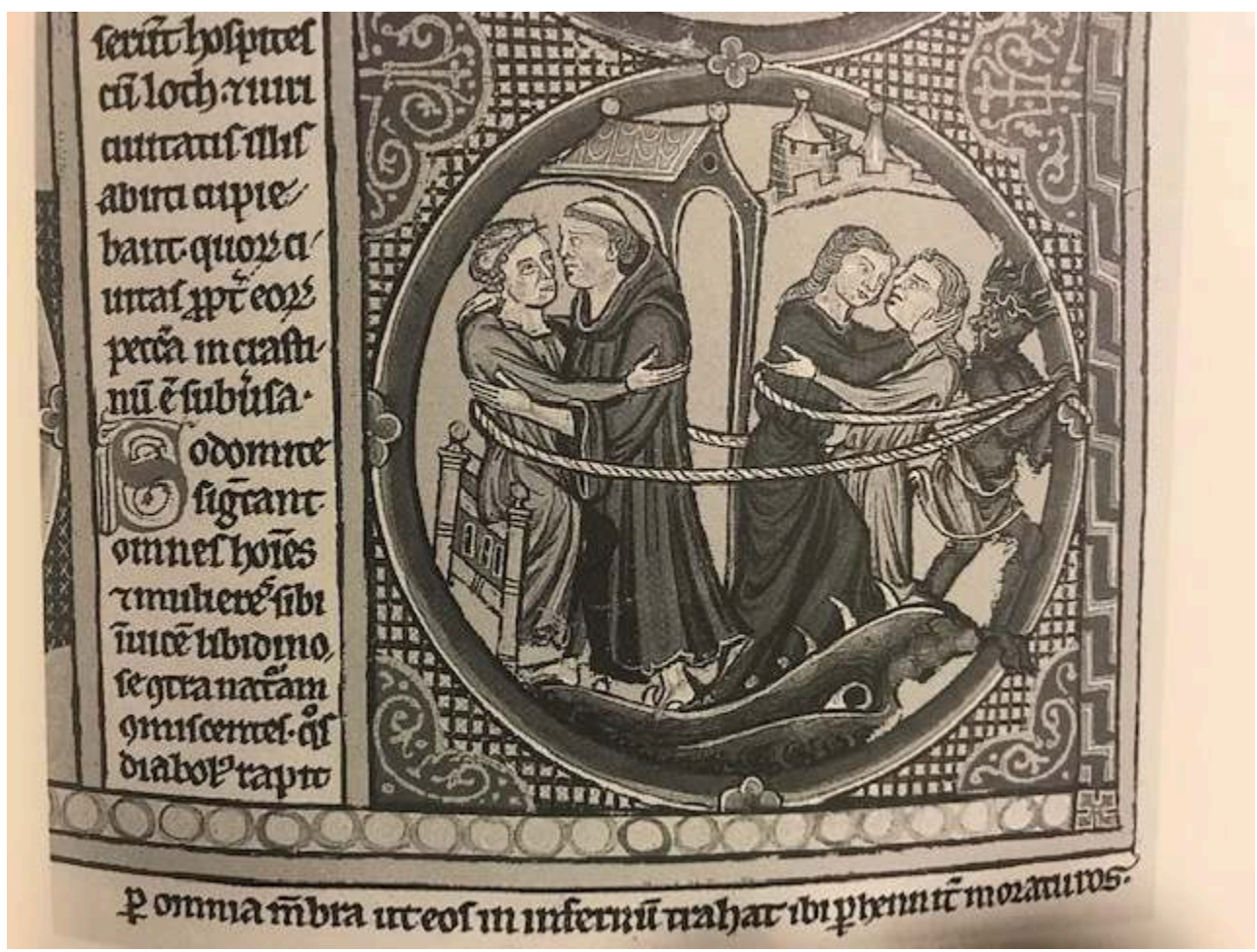

Bible moralisée, paris vers 1230, Toledo, Tesoro de catedral ms 1 fol. 14r ed. Moleiro

13 Mais la perspective de Mills est claire, il s'agit moins de chercher où étaient les lesbiennes que les transgenres sous la plume d'Hildegarde de Bingen, les catégories telles que l'identité de genre ou l'orientation sexuelles sont de notre époque, et comme le disait Halperin, le fait qu'elles prennent une telle importance dans la vie sociale est relativement récent. Pourtant il serait tout aussi faux de penser que si les lesbiennes n'existaient pas comme elles peuvent exister aujourd'hui, tout le monde était hétérosexuel. Les pratiques et les expériences existent tous de multiples facettes si on 
les cherche. Suivant Jack Halberstam, Mills propose de poser un regard transgenre, au sens littéral du terme (p. 98).

Et toutes les fois où se pose la question de l'attirance homoérotique, une question de genre se pose de la même façon, pour Eugénie, devenue le moine Eugène et agressé sexuellement par la lubrique Melancie, à Vezelay, comme pour Iphis et Ianthe, dans l'Ovide moralisé, imaginés comme un couple courtois quand le texte parle de deux femmes, dont l'une porte un vêtement masculin ${ }^{26}$.

\section{Romanité et amour entre hommes}

Mills use de la littérature romaine moralisée, pour mesurer les effets de l'interprétation chrétienne des relations entre hommes. Orphée apparaît comme le premier sodomite mythologique. Après la mort d'Euridice, il choisit d'aimer les hommes. Ce choix est défini comme "contre nature ", comme l'étaient les amours d'Iphis et Ianthé, de Pasiphae et du taureau, de Jupiter et Ganymède. Mais la gynophobie chrétienne permet une spiritualisation inattendue : se tourner vers les hommes peut être une volonté de se tourner vers l'amour spirituel. Comme Dieu aime tous les hommes, l'amour de Dieu : "a fera e devin plaisir» (p. 144). Cette interprétation n'est pas univoque, et les multiples versions postérieures de la mort d'Orphée étaient souvent lues comme liées à la punition de ses crimes sexuels.

Une série iconographique aborde plus spécifiquement cette question tout en étant omniprésente à dans l'art roman : l'enlèvement de Ganymède. Jean Wirth cherchait à montrer que les églises non encore réformées, et marquées par une moindre pression de la morale en matière sexuelle multipliaient les images de nus masculins facilement interprétables comme érotiques, comme à Saint Pierre de Mozat, exemple qui n'est pas cité par Mills ${ }^{27}$. Wirth interprète des références à la antiquité " pédéraste ", là où JeanClaude Bonne, Jérôme Baschet et Pierre-Olivier Dittmar préféraient interpréter un recours savant à un référentiel romain ("romanité»), présent des chapiteaux corinthiens aux victoires ailées ${ }^{28}$. Je critiquerais pour ma part les catégories exogènes de Jean Wirth, mais j'aimerais inviter Bonne Baschet et Dittmar à ne pas s'abstenir de commenter le goût pour le nu masculin dans les églises auvergnates.

Fig. 7

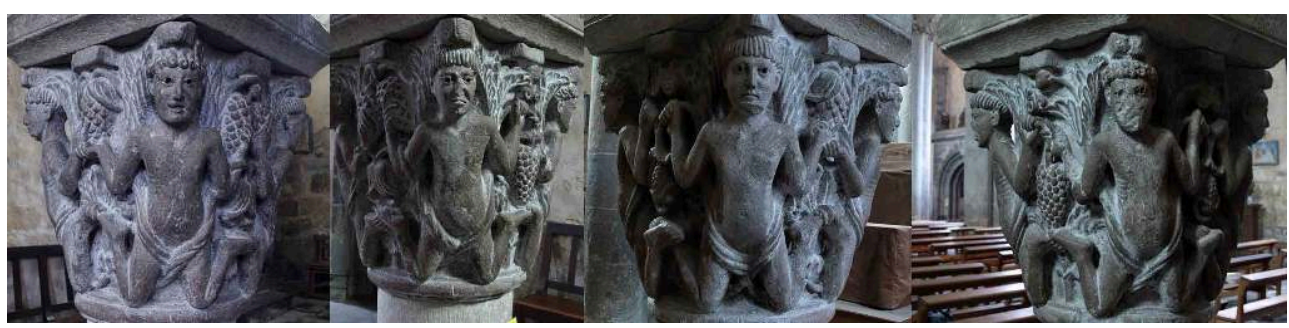

Saint Pierre de Mozat, c. 1080, Auvergne

17 Mills montre l'ambivalence des enlèvements de Ganymède. À Vézelay, Ganymède, dont un Jupiter-Aigle tord le corps en l'arrachant de terre, est terrifié : ce qui lui arrive pourrait être le châtiment des pêcheurs sodomites dans l'au-delà, et un avertissement pour le monde. 
Fig. 8

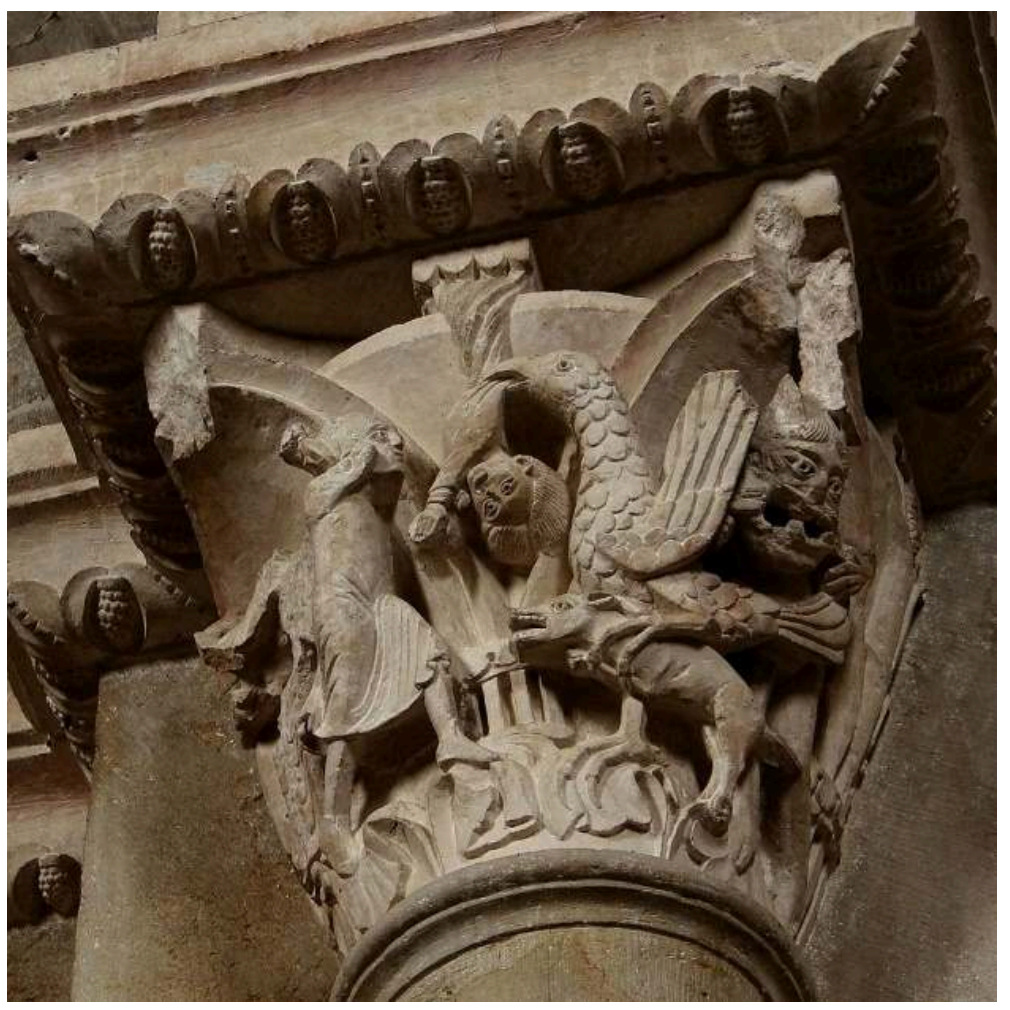

Église Sainte-Marie-Madeleine de Vezelay, l'enlèvement de ganymède, chapiteau de la nef, v. 1096-1106

18 Si l'image dans l'église de Vézelay ne présente aucune ambiguïté de sensualité, l'iconographie elle-même comme le montre Mills se prête à différentes interprétations. Au contraire, dans Ovide moralisé (v. 1317-1318), Ganymède devient Jean l'évangéliste, le préféré du Christ qui le tient contre son cœur. Le sens du rapt de Ganymède est celui d'une ascension divine (p. 93). 
Fig. 9

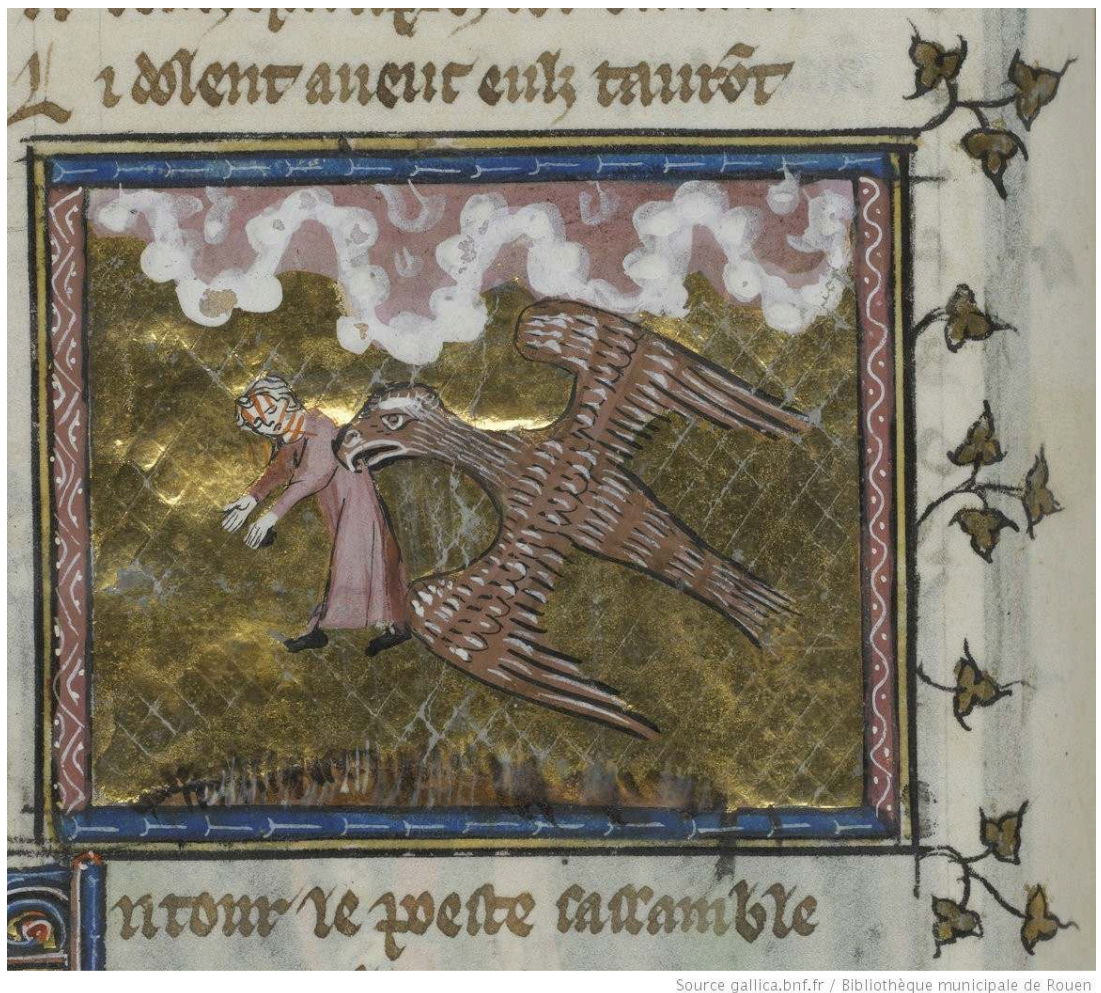

Miniature in Ovide moralisé . Paris, circa 1315- 28 . Rouen, Biblio thèque municipale, MS 0.4 (1044), fol. 250v (detail). Collections de la Bibliothèque municipale de Rouen. Photography C. Lancien; Y. Communeau.

19 Ganymède est jeune, non pas comme un éphèbe mais comme une âme emportée dans l'au-delà. Selon Mills, dans l'art de cette époque, il ne faut pas penser les thèmes sexuels comme des notions à bannir du côté des horreurs impossibles à mentionner. Si la question de la répression de la sexualité et de sa régulation étaient dans tous les esprits, la sexualité (y compris chaste) participait à la translation du plaisir charnel en un plaisir spirituel, notions pour lesquelles Mills convoque les concepts de subsitutio et translatio (p. 235). Il convoque l'idée de «contre-érotisme» de Virginia Burrus, historienne queer du christianisme qui avait interprété la jouissance (avec une bonne dose d'anachronismes empruntée aux relectures masochistes des $\mathrm{XIX}^{\mathrm{e}}$ et $\mathrm{XX}^{\mathrm{e}}$ siècle) dans l'hagiographie tardo-antique et son appétence pour la macération corporelle ${ }^{29}$.

\section{Marginalia et derrières}

Mills donne du sens à l'omniprésence difficilement explicable de la " phénoménologie de l'anus ", dans les marginalia peuplées d'êtres ayant souvent un visage sur le derrière et se pénétrant avec diverses lances. Les images marginales des manuscrits longtemps interprétées par l'histoire de l'art comme des fantaisies dépourvues de sens, sont désormais perçues comme des images sociales, les marges des images se mettant au diapason des marges du monde, et en présentant la face inversée, présente et absente ${ }^{30}$. 
Fig. 10

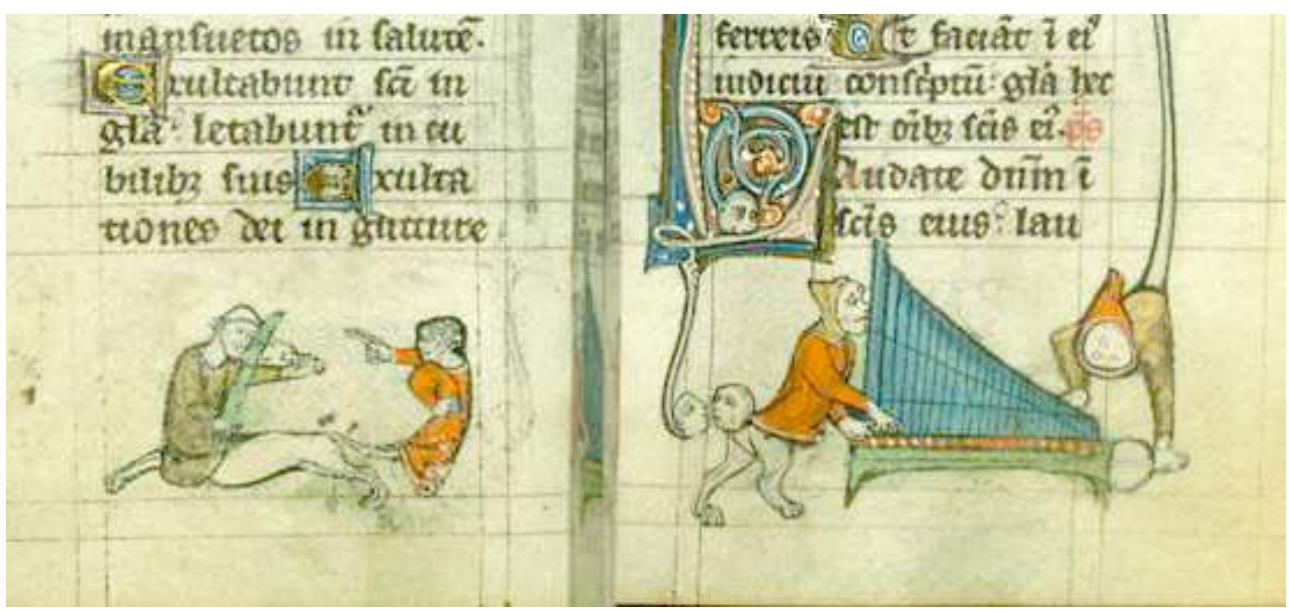

Walter Art Museum Ms W.88, fol 63v-54 (c.1300-1325)

$\mathrm{Au}$ Moyen-Âge, l'anus en tant que signe fonctionnait de manière variable - selon le contexte et le lieu - comme un dispositif apotropaïque, comme un motif carnavalesque de renversement des rôles et du monde à l'envers, ou comme une insulte diffamatoire (comme dans la pratique du "mooning"). Mais il fonctionne dans de tels contextes principalement comme un signe de pouvoir plutôt que de vulnérabilité - une extension du phallus que l'on voit parfois s'étendre depuis le corps du porteur de fesses ${ }^{31}$. (p. 88)

Sans rien retirer de la polysémie des derrières médiévaux, souvent négatifs, mais aussi protecteurs, injurieux mais propagateurs d'un souffle vital, Mills insiste sur le fait que l'anus est présenté dans les images comme un signe de pouvoir plutôt que de vulnérabilité, une manière d'interagir avec le monde, potentiellement grotesque, mais jamais faible.

Fig. 11

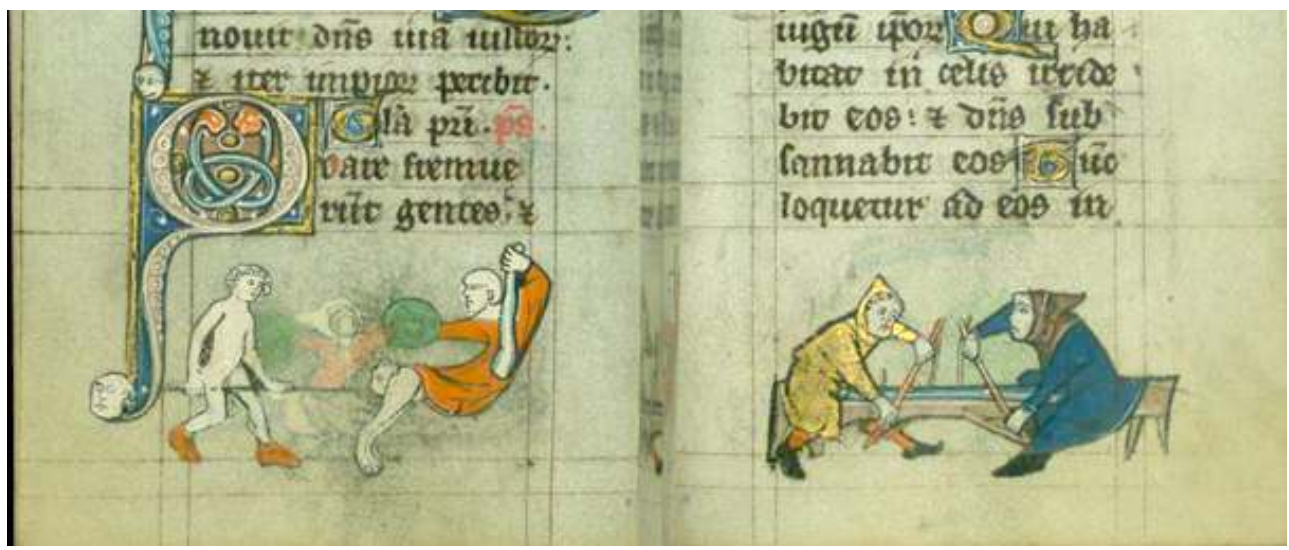

Walter Art Museum Ms W.88, fol 72v (c.1300-1325)

Ces images entretiennent un certain rapport avec le péché de sodomie, et le fait que les pénétrations anales occupent une place importante dans les tortures infligées aux condamnés de l'enfer en est un signal. 


\section{Le pouvoir érotique des images médiévales}

Fig. 12

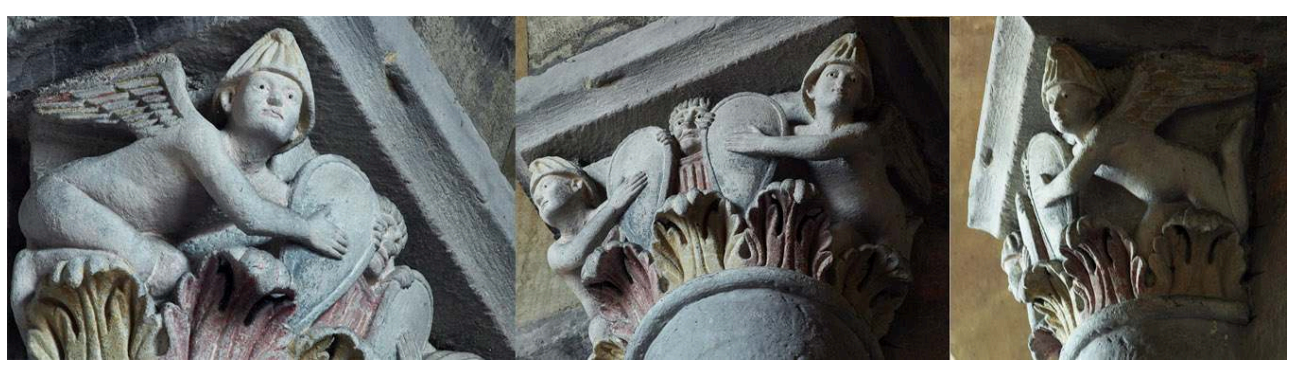

Saint Pierre de Mozat, c. 1080, Auvergne, victoires ailées tenant un bouclier.

Gahom-Alhoma sexuelle. Il est donc crucial de distinguer ce que l'on peut déterminer des intentions du ou de la peintre et de l'émotion particulière des personnes qui le reçoivent à différentes époques, et du contexte de réception de chaque image. Une Bible ayant pour but l'édification morale ne sera pas regardée de la même manière qu'une sculpture urbaine. Les sculptures dans une abbatiale, regardées uniquement par des hommes pétris de culture antique (Mozat) ne seront pas vues de la même façon qu'un livre d'heures aux mains d'un aristocrate réputé pour ses frasques sexuelles (Jean de Berry et les frères Limbourg). Le livre de Mills a le grand intérêt de poser une des premières pierres à un domaine historiographique excessivement lacunaire : il s'inscrit dans une tradition déjà riche d'études sur les sexualités médiévales, dans une perspective d'études LGBTQI+ en constante évolution depuis les années 1980, mais ne dispose que de peu de travaux en histoire de l'art. C'est donc toute l'histoire visuelle de l'homoérotisme qui demande à se construire. La piste iconographique permet au moins de supputer que la personne à l'origine de l'image pouvait avoir l'intention de représenter une scène sexuelle. Le péché de Sodome, l'enlèvement de Ganymède, la métamorphose d'Iphis et Ianthé font partie des quelques motifs qui renvoient explicitement à une relation d'amour entre personnes de même genre. Elles constituent ainsi la matrice à partir de laquelle une grammaire des images « homosexuelles » peut se dessiner.

Une piste de prolongation tient à travailler une histoire des gestes érotisés. Les étreintes et les gestes d'amour que l'on observe dans les Bibles moralisées sont très proches qu'il s'agisse de couples hétéro- ou homosexuels. La main se pose sur la hanche, les corps se rapprochent et la main se pose sur le menton. Ce geste a une signification très particulière parce qu'il décrit systématiquement une relation courtoise, et se retrouve dans de nombreuses images chevaleresques décrivant sans conteste des scènes amoureuses. Par contraste un baiser sur la bouche, explicitement érotique pour nos contemporains, ne saurait être compris comme un geste érotique à cette époque, car le baiser était utilisé à la fois dans le cadre du baiser de paix après la communion, et pour sceller une alliance politique, probablement sans aucune allusion sensuelle malgré les débats nombreux autour des baisers de paix entre rois ${ }^{32}$.

L'examen de ces images requiert donc se de débarrasser des fausses évidences concernant une histoire du corps et des gestes ${ }^{33}$. Un baiser sur la bouche ne veut rien dire d'autre qu'un geste bien codifié, une main sur le menton révèle un érotisme

Images Re-vues, 17 | 2020 
autrement plus suggestif. Mills analyse longuement cette bible moralisée devenue à tel point iconique qu'elle sert souvent à montrer les seuls homosexuels du Moyen Âge torturés par des démons. Son hypothèse est que l'image démontre à la fois un intérêt renouvelé pour la question de la sodomie à cette époque dans l'entourage royal qui produit ces Bibles moralisées, et qu'il s'agit d'image de translation, une transition par rapport au modèle «naturel » (p. 31). Parce que le livre s'inscrit dans un champ en plein essor, la question du désir, celle de la répression des sexualités, ou celle des images anales semblent apporter de la confusion et peinent à proposer une histoire continue. En espérant que des dossiers nouveaux viendront étayer une nouvelle philologie de l'amour médiéval et la grammaire des gestes érotiques, la perspective de Mills, inspirée par des auteurs fondateurs des études gaies et lesbiennes, queer, et trans, permet de penser les sexualités comme un lieu d'observation d'une séries d'images romanes et gothiques, complexifiant les définitions des personnes, des actes et des passages entre monde spirituel et charnel.

\section{NOTES}

1. Jérôme BASCHET, La civilisation féodale: de l'an mil à la colonisation de l'Amérique, Paris, France, 2018.

2. Robert MILLS, Seeing Sodomy in the Middle Ages, University of Chicago Press, London and Chicago, 2015.

3. John BOSWELL, Christianisme, tolérance sociale et homosexualité : les Homosexuels en Europe occidentale des débuts de l'ère chrétienne au XIVe siècle, Paris, Gallimard, 1985.

4. Damien BoQUET, "L'amitié comme problème au Moyen Âge ", dans Une histoire au présent. Les historiens et Michel Foucault, CNRS Editions, Paris, 2013, p. 59-81.

5. Frédérique LE NAN, «Voies du désir et vocalité désirante dans les chansons de femmes d'oc et d'oïl (XII ${ }^{\mathrm{e}}$ et XIII ${ }^{\mathrm{e}}$ siècles) ", Le désir : Or se cante, or se conte, Revue des langues romanes, $\mathrm{CXVII}, \mathrm{n}^{\circ} 2$, 2014, p. 381-402.

6. Alain CORBIN, Les filles de noce: misère sexuelle et prostitution, XIX ${ }^{e}$ siècle, Paris, France, 2015.

7. Jean Claude Schmitt a fait la critique des biais de cette histoire pratiquante: Jean-Claude scHMiTT, « Une histoire religieuse du Moyen Âge est-elle possible? », dans F. LEPORI, Fr. SANTI (éd.), Il mestiere di storico del medioevo, Solète, 1994, p. 73-83.

8. ID., «Christianisme et homosexualité. A propos du livre de John Boswell », dans Le Débat, 10, mars 1981, p. 144-160.

9. David M. HALPERIN, One hundred years of homosexuality: and other essays on Greek love, New York, Etats-Unis d'Amérique, Royaume-Uni de Grande-Bretagne et d'Irlande du Nord, 1990.

10. Le livre de Louis-Georges Tin, reprenant l'idée d'une émergence de l'hétérosexualité au XII ${ }^{\mathrm{e}}$ siècle et avec la littérature courtoise, a été critiqué par Didier Lett réfutant l'idée de culture ou d'orientation sexuelle pour cette période au profit d'actes sexuelles licites ou illicites (ces derniers pouvant concerner des hommes, des femmes, ou des hommes avec des femmes). LouisGeorges TIN, L'invention de la culture hétérosexuelle, Paris, France, 2008 ; Didier LETT, « Louis-Georges Tin, L'invention de la culture hétérosexuelle. Paris, Éditions Autrement (Collection Mutations/ Sexe en tous genres 249), 2008, 208 pages », Clio. Femmes, Genre, Histoire, 31, 2010, p. 287-290. 
11. Matt cooK, A Gay History of Britain: Love and Sex Between Men Since the Middle Ages, Oxford; Westport, Conn, 2007.

12. Michel Foucault, Histoire de la sexualité IV, Les aveux de la chair, Paris, France, 2018.

13. Damien BOQUET, L'ordre de l'affect au Moyen Âge. Autour de l'anthropologie affective d'Alfred de Rievaulx, Caen, 2005 ; BOQUET, « L'amitié comme problème au Moyen Âge... », art cit.

14. Pour ces analyses, je me permets de renvoyer à mon article «Jérôme de Stridon porte une robe, visualité du genre d'après une image des belles heures du duc de Berry par les frères Limbourg (104-1408) », dans L'Art du paraître : les apparences de l'humain, de la Préhistoire à nos jours, rencontres internationales d'archéologie et d'histoire, Nice, à paraître en 2020.

15. Pour cette analyse des rapport entre l'amour pour l'art de Jean de Berry et sa sexualités, Mills s'appuie sur: Michael CAMILLE, Adrian RIFKIN (éd.), Other objects of desire: collectors and collecting queerly, Oxford, UK, Royaume-Uni de Grande-Bretagne et d'Irlande du Nord, 2001, p. 15.

16. Ruth Mazo KARRAS, Sexuality in the Middle Ages. Doing Unto Others, New York, 2005.

17. Liber Gomorrhianus, Opuscule VII, Patrologie Latine publiée par l'abbé Migne, tome CXLV [145], colonnes 159-190.

18. Voir la synthèse de Didier Lett dans S. Steinberg (dir.), Une histoire des sexualités, PUF, Paris, 2018 p.133-134 et " Le 'vice sodomite' au Moyen Âge. Contribution à une histoire des homosexuels", dans Homosexualité et traditions monothéistes. Vers la fin d'un antagonisme ?, Rémy BETHMONT et Martine GROSS (dir.), Labor et fides, Genève, 2017, p. 247- 262.

19. Didier LETT, «

20. Jean WIRTH, L'image à l'époque romane, Paris, France, 1999, p. 165 sqq.

21. Ruth Mazo KARRAS, Sexuality in medieval Europe. Doing unto others, New York/Londres, Routledge, 2005.

22. Parmi les rares condamnations on trouve celle Katherina Hetzeldorfer en 1477 pour actes sexuels avec godemiché en bois et cuir. Elle est condamnée à la noyade dans le Rhin, ses partenaires, qui témoignent avoir été passives sont relaxées, cité par Didier Lett dans Sylvie STEINBERG et alii, Une histoire des sexualités, Paris, France, 2018.

23. François GANGON, Le Corrector sive Medicus de Burchard de Worms (1000-1025): présentation, traduction et commentaire ethno-historique, Mémoire de maîtrise en histoire, soutenue à l'Université de Montreal, 2010.

24. J. HALBERSTAM, In a queer time and place: transgender bodies, subcultural lives, New York, Etats-Unis d'Amérique, 2005.

25. Le chapitre 1 provient partielement de "Seeing Sodomy in the Bibles moralisées" in Speculum 87, no. 2 (2012): 413- 68. Et le chapitre 5 reprend" Gender, Sodomy, Friendship, and the Medieval Anchorhold," Journal of Medieval Religious Cultures 36, no. 1 (2010): 1- 27 et "Acts, Orientations and the Sodomites of San Gimignano," dans Sex Acts: Practice, Performance, Perversion and Punishment in Early Modern Italy , ed. Allison Levy (Farnham, UK: Ashgate, 2010), 195- 208.

26. C. MAILLET, « Des seins de moine à Vézelay. Eugène-Eugénie, nouvelle image transgenre au xiie siècle ", Gradhiva. Revue d'anthropologie et d'histoire des arts, 28, 2018, p. 220-243.

27. "Même le grand saint Anselme écrivit à de jeunes moines les lettres enflammées à de jeunes moines où il exprima tous les tourments de l'amour. Il est probable que de tels attachements aient été platoniques chez les plus pieux, mais cela ne change rien à notre propos. La sensibilité esthétique à la beauté des garçons est très vive dans le clergé de l'époque et s'exprime sans réticence au grand jour, tout en étant critiquée. On en vient même à se demander si l'étalage systématique de ces goûts dans la somptueuse église de Mozat n'est pas une des raisons qui conduisirent l'évêque de Clermont à mettre la vénérable abbaye sous la tutelle de Cluny ». WIRTH, L'image à l'époque romane..., op. cit. à la note 18, p. 168.

28. «La puissante inspiration antiquisante de la sculpture de Mozat ne signifierait pas tant une tolérance à l'égard du paganisme et de la chair qu'une référence à un passé romain prestigieux, 
auquel est associé le fondateur supposé de l'abbaye, le sénateur romain Calminius. L'atmosphère romanisante créée par le sculpteur serait une manière d'hommage à l'époque du fondateur, venant exalter l'ancienneté prestigieuse de l'abbaye " Jérôme BASCHET, Jean-Claude BONNE, PierreOlivier DITTMAR, "Chapitre II - Saint-Pierre de Mozat: entre dignité du monde terrestre et harmonies cosmologiques ", Images Re-vues. Histoire, anthropologie et théorie de l'art, Hors-série 3, 2012. https://journals.openedition.org/imagesrevues/1664

29. Virginia BURRUS, La vie sexuelle des saints: l'art érotique de l'hagiographie ancienne, Montrouge, France, 2010.

30. Michael CAmille, Images dans les marges: aux limites de l'art médiéval, Paris, France, 1997 ; JeanClaude sснмітт, «L'univers des marges », dans Le Moyen Âge en lumière, 2002, p. 328-361.

31. "In the Middle Ages, the sign of the anus functioned variously- depending on context and location - as an apotropaic device, as a motif of carnivalesque role reversal and the world upside down, or as a defamatory insult (as in the practice of "mooning"). But it operates in such settings primarily as a sign of power rather than vulnerability - an extension of the phallus sometimes seen extending from the bum- bearer's body", R. MILLS, op cit., p. 88, traduction personnelle.

32. SCHMITT, L'univers des marges..., op. cit.

Boswell interprétait le baiser entre Richard Cœur de Lion et Philippe Auguste comme une histoire romantique. Nul ne saura si elle est advenue entre ces deux personnes dont les secrets restent cachés pour l'histoire. Il est en revanche probable que le roi ait été condamné pour d'autres relations avec des hommes, car il fit pénitence pour bougrerie. En revanche la démonstration publique de leurs baiser et de leur amitié s'étendant jusqu'à partager le même lit est un témoignage politique d'alliance. Jean FLORI, Richard Coeur de Lion: le roi-chevalier, Paris, France, 2000, p. 234-253.

33. Qui avait été brillamment exposée dans sa différence anthropologique profonde par JeanClaude schмiтt, dans La raison des gestes dans l'Occident médiéval, Paris, France, 1990.

\section{RÉSUMÉS}

La question de l'homoérotisme des images médiévales pose question dans la mesure où les images sont majoritairement produites dans le cadre de l'institution ecclésiale, qui condamne idéologiquement la sexualité entre personnes de même genre. Les pistes iconographiques, et les méthodes d'analyses d'images contextuelles permettent de mettre en perspective des séries de la question du nu à celle de l'analité. Cette note de lecture de l'ouvrage de Robert Mills propose une synthèse critique et prospective des publications récentes dans ce domaine.

The question of the homoeroticism of medieval images arises problems inasmuch as the images are mostly produced within the framework of ecclesial institution, which ideologically condemns sexuality between people of the same gender. The iconographic tracks, and the methods of analysis of contextual images allow us to put into perspective series, from the question of the nude to anality. This review of Robert Mills' book proposes a critical and prospective synthesis of recent publications in this field. 
INDEX

Keywords : homoeroticism, homosexuality, Middle Ages, moralized Bible, Brothers Limburg, marginalia

Mots-clés : homoérotisme, homosexualité, Moyen Âge, Bible moralisée, Frères Limbourg, marginalia

\section{AUTEURS}

\section{CHLOÉ CLOVIS MAILLET}

C. Maillet est historien'ne, auteur'e d'une thèse de l'EHESS sur la parenté hagiographique médiévale sous la direction de Jean-Claude Schmitt (publiée chez Brepols publishers en 2014), et spécialiste des questions de genre et de parenté dans la culture visuelle et cléricale médiévale. Après une bourse post-doctorale au musée du quai Branly en 2015-2016, une bourse de recherche à la coopérative de l'ESACM avec L. Hervé, enseigne à l'ESAD TALM Angers, à l'UCO Angers.

Membre du comité de rédaction d'Images re-vues, a publié des articles dans Gradhiva, Mediévales, Terrain et de nombreux ouvrages collectifs. Les Genres Fluides, de Jeanne d'arc aux saintes trans est en 2020 aux éditions Arkhê. Coordonne le Mois du genre entre l'ESAD TALM et l'université d'Angers depuis 2019. 